\title{
Multi-modal leaky Lamb waves in two parallel and immersed plates: Theoretical considerations, simulations, and measurements
}

\author{
Pierre Kauffmann, ${ }^{1, a)}$ Marie-Aude Ploix, ${ }^{2}$ Jean-François Chaix, ${ }^{2}$ Catherine Potel, ${ }^{3}$ \\ Cécile Gueudre, ${ }^{2}$ Gilles Corneloup, ${ }^{2}$ and François Baque ${ }^{1}$ \\ ${ }^{1}$ Commissariat à l' Énergie Atomique et aux Énergies Alternatives Cadarache, Direction de l'énergie \\ nucléaire / Département de Technologie Nucléaire / Service de Technologie des Composants et des Procédés, \\ Laboratoire d'Instrumentation, Systèmes et Méthodes, 13108 Saint Paul Lez Durance, France \\ ${ }^{2}$ Aix-Marseille Université, Centre National de la Recherche Scientifique, Centrale Marseille, Laboratoire de \\ Mécanique et d'Acoustique, Waves and Imaging Group, Institut Universitaire de Technologie, Génie \\ Mécanique et Productique, 413 Avenue Gaston Berger, 13625 Aix en Provence Cedex 1, France \\ ${ }^{3}$ Laboratoire d'Acoustique de l'Universite du Mans, Unité Mixte de Recherche, Centre National de la \\ Recherche Scientifique 6613, Avenue Olivier Messiaen, 72085 Le Mans Cedex 9, France
}

(Received 20 April 2018; revised 31 January 2019; accepted 1 February 2019; published online 22 February 2019)

Leaky Lamb waves have the potential to be used to perform non-destructive testing on a set of several parallel and immersed plates. Short-time Fourier transform and two-dimensional Fourier transform have both been successfully used to measure the propagation properties: phase and group velocity, and leaky attenuation. Experimental measurements were validated by comparison between theory, experimentation and finite-element simulations (using COMSOL MULTIPHYSICS ${ }^{\circledR}$ software) in the case of one immersed plate in water. These signal processing techniques proved to be efficient in the case of multi-modal propagation. They were applied to two immersed plates to identify the leaky Lamb mode generated in the second plate. Dispersion curves of the system composed by two immersed and parallel plates are computed. When plates have the same thickness, leaky Lamb modes propagate from the first to the second plate without any mode change, with the apparent attenuation being weaker in the second plate. Considering that the second plate is continuously supplied in energy by the first one, an energy-based model is proposed herein to estimate the apparent attenuation in the second plate. Despite our extremely simplifying assumption, this model proved to be in good agreement with both finite-element modelling and experimentation.

(C) 2019 Acoustical Society of America. https://doi.org/10.1121/1.5091689

[NJK]

Pages: 1018-1030

\section{INTRODUCTION}

The sodium-cooled fast reactor concept (SFR) has been chosen for the fourth generation of nuclear power plants in France. With liquid sodium as the coolant, improvement of in-service inspection and repair (ISI\&R) has been identified as a major issue for the Advanced Sodium Technological Reactor for Industrial Demonstration (ASTRID) project (Baqué et al., 2015). Well adapted to this harsh environment, ultrasonic solutions are being studied for the inspection of the main vessel and different parts of ASTRID. The immersion of specific ultrasonic transducers in liquid sodium is currently being investigated (Baqué et al., 2012). However, it appears that inspection operations performed with a transducer located outside the main vessel (in the inter-vessel gap filled with gas) have shown promising potential (Baqué et al., 2011; Corneloup et al., 2011). The objective is not only to perform non-destructive testing (NDT) of the main

\footnotetext{
a) Also at: Aix-Marseille Université, Centre National de la Recherche Scientifique, Centrale Marseille, Laboratoire de Mécanique et d'Acoustique, Waves and Imaging Group, IUT GMP, 413 Avenue Gaston Berger, 13625 Aix en Provence Cedex 1, France. Electronic mail: pierrekauffmann@ hotmail.fr
}

vessel itself, but also of structures inside this vessel which are immersed in liquid sodium. The diameter of the main vessel and its internal baffles is quite large (about $16 \mathrm{~m}$ ), which justifies locally approximating their shape by two parallel plates (Fig. 1). The challenge is then to propagate ultrasonic waves from one plate to another through the liquid so as to inspect them. Leaky Lamb waves appeared as a good candidate for such inspection purposes.

NDT will be performed by analyzing the characteristics of ultrasonic echoes reflecting back from structures immersed in liquid sodium (time of flight and amplitude). Substantial changing in these structures (such as flaws or cracks) should produce the echoes (Alleyne and Cawley, 1992b; Castaings et al., 2002). To perform such NDT, knowledge of the guided wave velocity and attenuation is essential as these parameters determine the acoustic reemission between immersed plates. In the case of a steel plate immersed in water, it has been shown that variations induced by the surrounding fluid on velocities are negligible (Chimenti and Rokhlin, 1990). However, attenuation increases because of the reemission of bulk waves in the fluid (Dayal and Kinra, 1989; Merkulov, 1964; Nayfeh, 1995; Nayfeh and Nagy, 1997; Viktorov, 2013): in this case, 


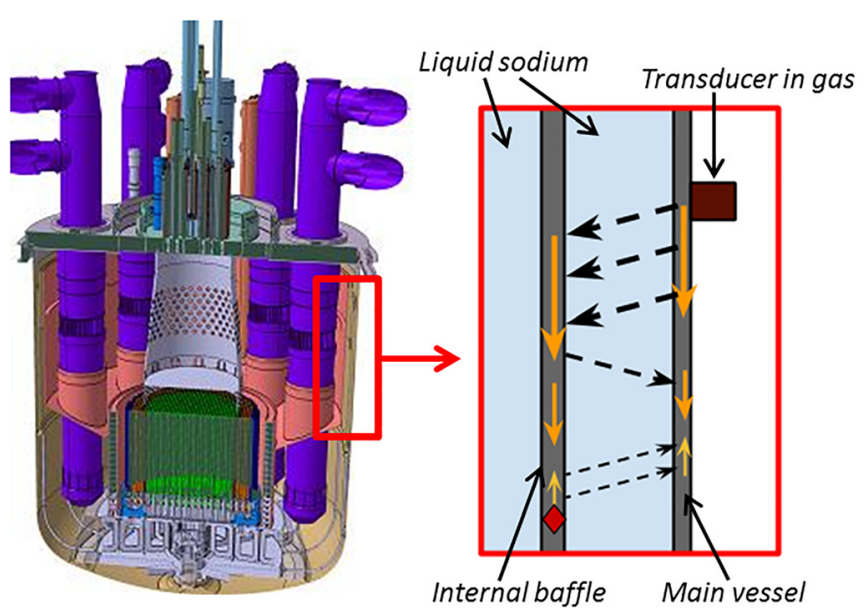

FIG. 1. (Color online) Cross section of the ASTRID reactor block indicating the area to be inspected (box with arrow) and a schematic view of this area indicating the expected ultrasonic path.

Lamb waves are referred to as leaky Lamb waves. We set out to measure this leaky attenuation because it is related to the creation and the supply of leaky Lamb waves in a second parallel plate. We will clearly distinguish two different kind of attenuation in this paper: the leaky attenuation and the attenuation due to the material. The leaky attenuation is due to the leakage of energy into a surrounding fluid and has been previously presented, and the attenuation due to the material is caused by scattering or by absorption in a viscoelastic material (Castaings et al., 2004; Chan and Cawley, 1998; Coquin, 1964; Minonzio et al., 2011).

Velocities of Lamb waves are well understood in the literature (Pavlakovic et al., 1997; Rose, 1999). Lamb modes propagate in a dispersive manner, which means that both phase and group velocities have to be considered (Kobayashi, 1994; Luis Dean-Ben et al., 2010) and their respective values depend on frequency. Being frequencydependent may result in the large spreading of wave packets. For NDT and local defect location, this spreading can be reduced by choosing a specific Lamb mode and a frequency where the rate of spreading is low (Wilcox et al., 2001b). Another technique would be to compensate the spreading afterwards by a post-processing technique: by $a$ priori knowledge of group velocity, each frequency component can be numerically retro-propagated and the original shape of the signal reconstituted at the time it was actually received (Wilcox, 2003). However, dispersive propagation complicates velocity and attenuation measurements (Kobayashi, 1994), leading to frequency methods that have been successfully used for the cases of a plate in air and in water. To measure phase velocity, the twodimensional Fourier transform (2D-FFT) has been widely used (Alleyne and Cawley, 1991; Harb and Yuan, 2015; Paget and Rehman, 2017): this method makes it possible to distinguish different Lamb modes in the frequency, wave number domain that overlap in time representation. The use of 2D-FFT to measure the attenuation (imaginary part of wave number) requires that the data be split into two equal parts and 2D-FFT to be performed on each part. Spectral amplitudes of both 2D-FFT are used to calculate the attenuation coefficient by taking the logarithm of their ratio, which requires the decrease to be exponential. This process is extensively explained and has been experimentally validated in Castaings et al. (2004) for the case of visco-elastic material. It seems that measurement of leaky attenuation has not yet been achieved using this technique for a plate in water. This paper shows that 2D-FFT is indeed very efficient for measuring attenuation in one immersed plate, but purposeless for a second parallel plate. Time-frequency techniques [short-time Fourier transform (STFT) and wavelet techniques] have been used to visualize the propagation of Lamb wave for a plate in air (Niethammer et al., 2000; $\mathrm{Xu}$ and $\mathrm{Hu}, 2017$ ) and less frequently in water (Takiy et al., 2017). However, even if these time-frequency techniques can separate each frequency component of the acoustic signal, they are inefficient in the case of Lamb modes propagating together at the same frequency and temporally overlapping. Temporal contributions of each frequency component need to be clearly distinct for each mode: this is easily done when Lamb modes can propagate far enough to separate their echoes, but this condition is hard to fulfil for leaky Lamb waves that are strongly attenuated. One reference has been found where the attenuation is measured with a time frequency technique in one immersed plate (Takiy et al., 2017), but so far there is no evidence of such measurement on a second immersed plate. Moreover, the use of STFT have not yet been validated for leaky attenuation measurement. This paper shows that the 2D-FFT and STFT techniques are complementary and in good agreement for one immersed plate, but also that measurement of attenuation with the 2D-FFT process is not applicable.

The angle incidence technique is widely used to generate leaky Lamb waves in an immersed plate: the targeted leaky Lamb mode is selected by the incidence of an acoustic beam generated by an immersed transducer or through an immersed wedge (Alleyne and Cawley, 1992a; Bertoni and Tamir, 1973; Kauffmann et al., 2018b; Nayfeh, 1995; Takiy et al., 2017; Viktorov, 2013; Wilcox et al., 2001b; Xu and Hu, 2017). It has been shown by Wilcox et al. (2001a) that some leaky Lamb modes are more excitable than others with this technique. This technique also leads to the excitation of several leaky Lamb modes together because of the beam-spreading angle, opening the door to multi-modal propagation (Alleyne and Cawley, 1992a). It is proving to be more of a challenge to measure velocities and attenuation in the case of multi-modal propagation: 2D-FFT seems to handle it well (Alleyne and Cawley, 1991), but the compatibility of the time frequency techniques has yet to be evaluated. We assess here the relevance of STFT in the case of multi-modal propagation of leaky Lamb modes.

Assessing a configuration of several parallel plates is quite innovative. Coulouvrat et al. (1998) worked on a trilayer solid/fluid/solid with no NDT application on any plate. A machined notch has been observed in a second and third parallel plate using the A0 mode for plates that were 2 and $3 \mathrm{~mm}$ thick at $1 \mathrm{MHz}$ (Baqué et al., 2011; Corneloup et al., 2011). It has also been shown that leaky Lamb waves seem to propagate from the first plate to the second for a system of two semi-immersed plates (Lindner 
et al., 2006; Schmitt et al., 2013). Steady state properties (reflection and transmission coefficient) of the multi-layer system water/plate/water/plate/water has already been investigated (Corneloup et al., 2011). However, the generation and propagation of leaky Lamb modes in a second plate has never been yet studied and characterized: this is achieved in this work.

This paper has two parts: The first part focuses on multi-modal propagation in one immersed plate: measurements of phase velocity, group velocity and leaky attenuation using two different techniques (2D-FFT and STFT) are discussed. The results of numerical simulations and experimentation are presented, and the use and implementation of the post-processing techniques for leaky Lamb wave measurements are validated in order to validate the theoretical leaky attenuation for one plate. In the second part, we apply the post-processing techniques validated before to the case of two parallel and immersed plates. A simple model based on the exchange of energy between plates (EBM) is presented and its limits are underlined. This model gives us insight into the apparent leaky attenuation in the second parallel plate that is different to that in the first plate. Finite-element simulations and measurements of leaky Lamb waves in the second plate confirmed this apparent attenuation and validated our theoretical model despite our extremely simplifying assumption.

\section{MULTI-MODAL PROPAGATION IN ONE IMMERSED PLATE}

The purpose of this section is to validate techniques to measure phase and group velocity and leaky attenuation in multi-modal propagation.

Since experiments in liquid sodium are complex and liquid sodium is quite similar to water from the acoustic point of view, experiments and simulations were performed in water at ambient temperature. For the sake of clarity, the same configuration is detailed in this paper and hereafter referred to as the "studied configuration": one (or two) stainless steel plate(s) $7.8 \mathrm{~mm}$ thick with an acoustic beam incident at $\theta_{i}=17.3^{\circ}$ generated by a $500 \mathrm{kHz}$ central frequency transducer (i.e., a product frequency thickness of $3.9 \mathrm{MHz}$ $\mathrm{mm}$ ) with an active diameter of $38.1 \mathrm{~mm}$.

\section{A. Theory}

Theoretical phase velocity and leaky attenuation are computed based on the equations first published in Merkulov (1964). These equations are recalled herein: (1) is for symmetric modes and (2) for antisymmetric modes. The contribution of water is included in the imaginary part: if this imaginary part is removed, the widely used equations for a plate in a vacuum can be retrieved,

$$
\frac{\tanh \left(\frac{\beta h}{2}\right)}{\tanh \left(\frac{\gamma h}{2}\right)}-\frac{4 k^{2} \beta \gamma}{\left(k^{2}+\beta^{2}\right)^{2}}+i \frac{\rho_{0} k_{t}^{4} \gamma \tanh \left(\frac{\beta h}{2}\right)}{\rho \sqrt{k_{0}^{2}-k^{2}}\left(k^{2}+\beta^{2}\right)^{2}}=0,
$$

$$
\begin{aligned}
& \frac{\tanh \left(\frac{\gamma h}{2}\right)}{\tanh \left(\frac{\beta h}{2}\right)}-\frac{4 k^{2} \beta \gamma}{\left(k^{2}+\beta^{2}\right)^{2}} \\
& +i \frac{\rho_{0} k_{t}^{4} \gamma}{\rho \sqrt{k_{0}^{2}-k^{2}}\left(k^{2}+\beta^{2}\right)^{2} \tanh \left(\frac{\beta h}{2}\right)}=0,
\end{aligned}
$$

with $i$ representing the imaginary unit, $h$ and $\rho$ the thickness and the density of the elastic plate respectively, $\rho_{0}$ representing the density of the surrounding fluid, $\beta=\sqrt{k^{2}-k_{T}^{2}}$, $\gamma=\sqrt{k^{2}-k_{L}^{2}}, k_{T}$ and $k_{L}$ representing the wave number of transverse and longitudinal waves in the plate, and $k$ the unknown wave number of the Lamb mode that propagates in the plate.

The solution for a plate in a vacuum was first computed and then used as an initializer to solve the immersed case with a Levenberg-Marquardt algorithm implemented in the MATLAB ${ }^{\circledR}$ software. The real part of $k$ was used to calculate the phase velocity plotted in Fig. 5(a), while the imaginary part was the leaky attenuation plotted in Fig. 6 and Fig. 7. This attenuation has been used in Sec. III B to calculate the apparent attenuation in a second parallel plate. Finally, the group velocity was calculated from the phase velocity (Rose, 1999) and is plotted in Fig. 5(b). As the leaky attenuation values are conditioned by the plate thickness, we plotted the attenuation multiplied by the plate thickness to overcome this issue and to standardize attenuation in the same way as in Merkulov (1964) and Wilcox et al. (2001a).

The following assumptions were applied: the plate was considered to be elastic, isotropic and homogeneous (allowing 2D projection) in stainless steel (a density of $7950 \mathrm{~kg} / \mathrm{m}^{3}$, and longitudinal and shear velocities of $5750 \mathrm{~m} / \mathrm{s}$ and $3150 \mathrm{~m} / \mathrm{s}$, respectively), with no attenuation due to absorption and scattering. Water was modelled with a density of $1000 \mathrm{~kg} / \mathrm{m}^{3}$ and a speed of sound equal to $1490 \mathrm{~m} / \mathrm{s}$. The viscosity of water was neglected. For comparison, dispersion curves with the viscosity of water taken into account can be seen in Takiy et al. (2017).

\section{B. Finite-element simulations}

The aim of this section is to validate the use of postprocessing techniques (2D-FFT and STFT) using finite-element models (FEM) with the COMSOL MULTIPHYSICS ${ }^{\circledR}$ software.

The FEM model was first validated with 2D simulations in frequency domain: the phase velocity and leaky attenuation reflected the theory perfectly. It can therefore be said that this finite-element model is relevant for studying leaky Lamb waves.

Next, the same model was used for simulations in the time domain in order to validate the post-processing techniques. The mesh was designed with rectangular elements with a maximum size of $\lambda_{\min } / 6$ in the plate and in water (with $\lambda_{\min }$ representing the smallest wavelength comprise in the broadband pulse of the Lamb waves or bulk waves that propagate in the plate and in the water, respectively). Leaky Lamb waves were excited on the left side of the plate by imposing their theoretical displacement profiles along the thickness (Viktorov, 2013) in the $X$ and 


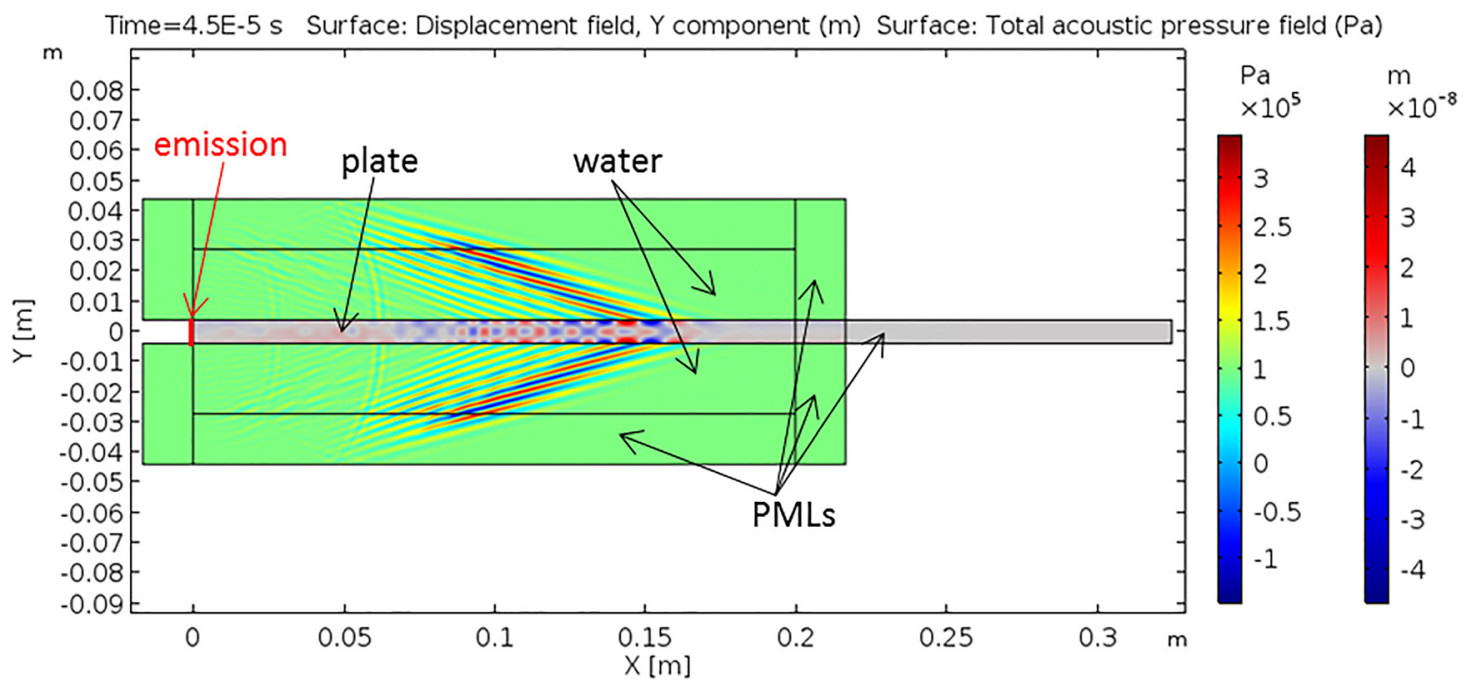

FIG. 2. (Color online) FEM simulation in the temporal domain of the leaky A1 mode around $3 \mathrm{MHz}$ mm. The pressure in water and the vertical displacements in the plate are both plotted.

$Y$ directions [a similar excitation technique was used in Castaings et al. (2004)]. In order to study and measure velocities of dispersive propagation, we used a signal with a broadband pulse: three sinusoid cycles windowed with a temporal Gaussian. Reflection at the end of the plate was considered null with the use of a perfectly matched layer (PML). PMLs are also used to bounds the water section and avoid any reflection that would create another leaky Lamb mode. Simulations were performed in $\mathrm{A} 0$ and $\mathrm{S} 0$ mode at $2 \mathrm{MHz} \mathrm{mm}$, with $\mathrm{A} 1$ around $3 \mathrm{MHz} \mathrm{mm}$ and $\mathrm{S} 1$ around $4 \mathrm{MHz} \mathrm{mm}$.

The leaky A1 mode around $3 \mathrm{MHz} \mathrm{mm}$ is plotted in Fig. 2. It can be seen the initial three-cycle signal has spread in space due to dispersive propagation.

Temporal signals were extracted every millimeter along the $200 \mathrm{~mm}$-length plate. These signals were then processed with 2D-FFT (to measure phase velocity and leaky attenuation) and with STFT (to measure group velocity and leaky attenuation). The final results are superimposed against the theoretical and experimental results in Fig. 4, and show very good agreement with theory. This validates the two post-processing techniques which can be applied to experimental data.

\section{Experimentation in water}

This section describes the experiments and discusses two spectrograms from STFT post-processing to illustrate multi-modal and dispersive propagation.

As shown in Fig. 3, we considered a single plate immersed in water. A transducer sent a pulse at a given central frequency and a chosen incident angle $\theta_{i}$ (measured perpendicular to the plate). A needle hydrophone scanned and recorded the transmitted field on a line parallel to the plate. This experiment was performed for the three central frequencies of three different transducers (corresponding to 0.86 , 1.95, and $3.9 \mathrm{MHz} \mathrm{mm}$ for the $7.8 \mathrm{~mm}$-thick plate) and their corresponding angle to each excitable leaky Lamb mode.

For the studied configuration (3.9 MHz mm), five Lamb modes propagated in the plate: A0, S0, A1, S1, and S2. Mode A1 was targeted with an incidence angle of $17.3^{\circ}$.
Temporal signals show propagation of at least two leaky Lamb modes that were attenuated too quickly for their echoes to be separated, which meant it was not possible to clearly identify them. For this reason, we used a time frequency technique to separate the contribution of each mode in the frequency range. Two examples of spectrograms given by a short-time Fourier transform algorithm are shown in Fig. 4. The results (shown as red dots in the figures) used to finalize the data processing were selected at the maximum amplitude for each frequency. Their arrival time gives the group velocity after linear regression, while their spectral amplitude gives the attenuation coefficient after exponential fitting. The final results are shown in Fig. 5 and Fig. 7.

Figure 4 shows that two packets distinctly propagate at $300 \mathrm{kHz}$ (i.e., $2.34 \mathrm{MHz} \mathrm{mm}$ ) and $500 \mathrm{kHz}$ (i.e., $3.9 \mathrm{MHz}$ $\mathrm{mm}$ ). Their arrival times are similar on the first spectrogram. They are strongly attenuated during their propagation due to the leakage of energy into the fluid: it proved difficult to

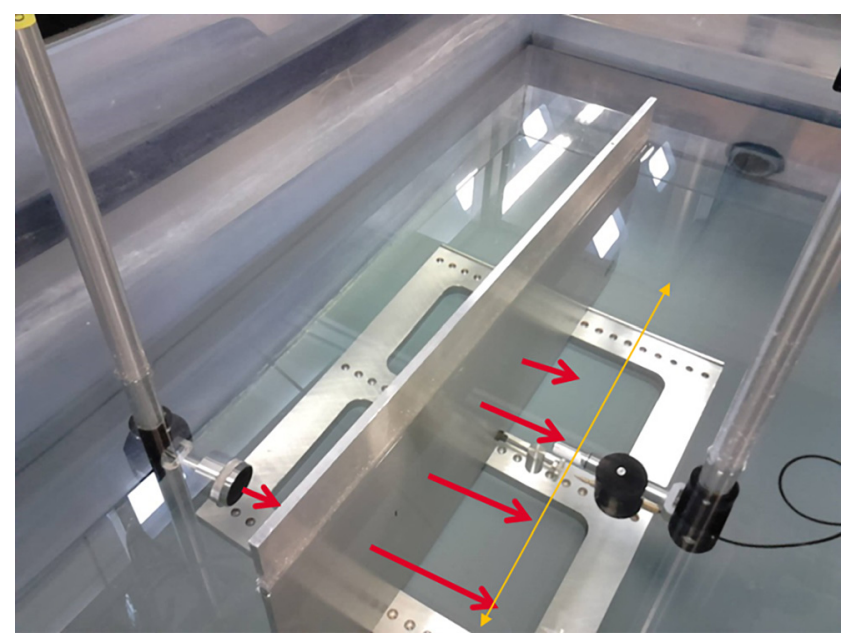

FIG. 3. (Color online) Test bench: a transducer sends bulk waves at a chosen incident angle $\theta_{i}$ onto a plate immersed in water $(7.8 \mathrm{~mm}$ thick and $700 \mathrm{~mm}$ long). A needle hydrophone scans the transmitted acoustic field on a line parallel to the plate (thin arrow). The bolds arrows symbolize the waves in water (incident and reemitted waves). 


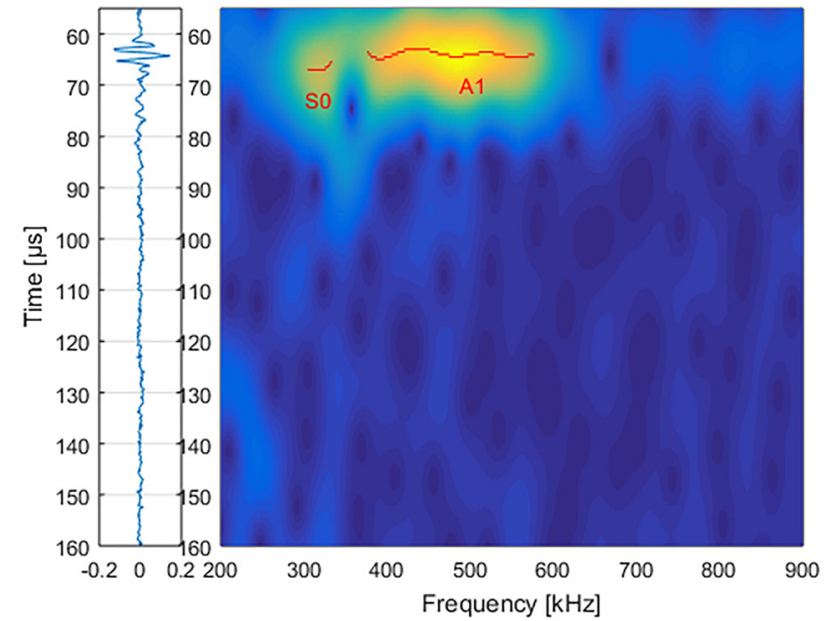

(a)

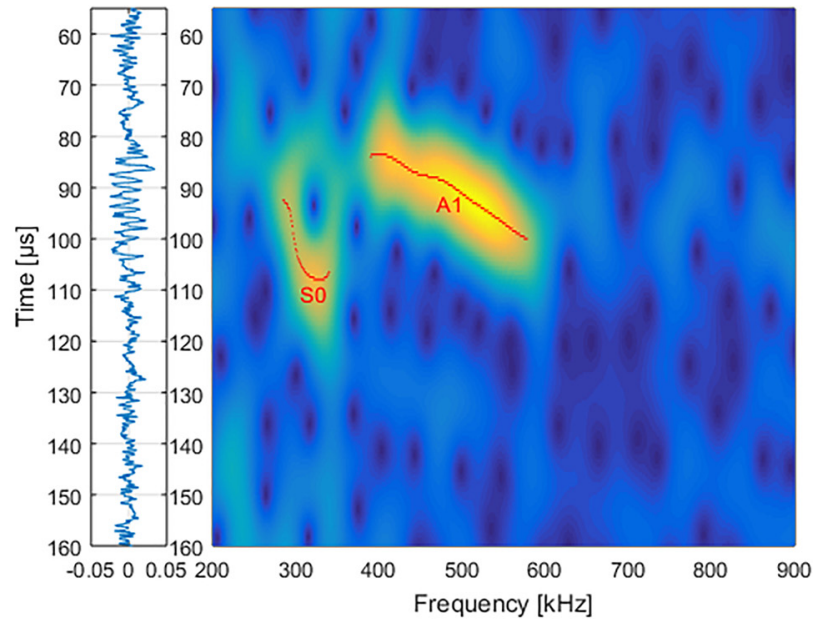

(b)

FIG. 4. (Color online) Spectrogram at the beginning of propagation (a) and after propagation of $80 \mathrm{~mm}$ (b) for the studied configuration. The temporal signal studied is plotted on the left side of each figure. Red dots indicate the data used to calculate the group velocity and attenuation.

observe them after $80 \mathrm{~mm}$ of propagation. However, for a propagation of $80 \mathrm{~mm}$, these two wave packets arrived at different times and each of their frequency components travelled at different velocities. These packets were identified as the S0 and A1 Lamb modes respectively once their group velocities were calculated. Figure 5(b) shows that the group velocity of S0 decreases and reaches a minimum at $2.3 \mathrm{MHz}$ $\mathrm{mm}$. This is also observed in the second spectrogram: lower frequencies of the S0 packet arrive earlier (i.e., faster) than the higher frequencies. The same observation is also valid for A1 around 3.9 MHz.mm.

There is no experimental observation of the S1 leaky Lamb mode because its reemission was almost null at $500 \mathrm{kHz}$ (see Figs. 6 and 7).

\section{Results and discussion}

This section presents the final results of phase and group velocity and leaky attenuation that were measured in FEM

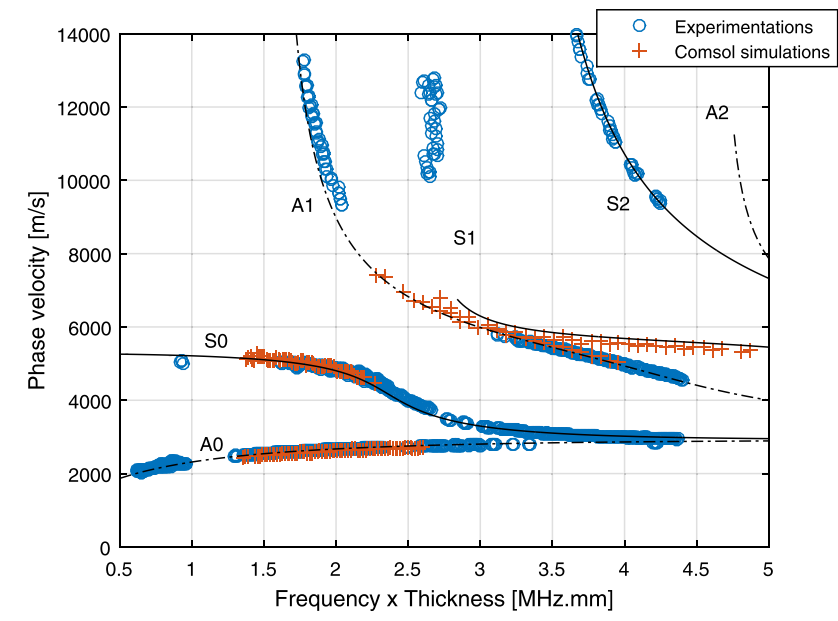

(a) and through experimentation. The efficiency of each postprocessing technique is discussed in detail.

Figure 5(a) compares the theoretical results with the experimental results (calculated by 2D-FFT) of the phase velocity: good agreement is observed. The biggest gap between theory and experimentation is $7 \%$ for $\mathrm{A} 1$ close to its cutoff frequency. 2D-FFT processing makes it possible to separate the Lamb modes in terms of the frequency and wave number, even when their echoes are not temporally distinct. As a result, all the experiments performed can be considered relevant and exploitable.

The comparison for group velocities is presented in Fig. 5(b). Good agreement with theory is observed, the worst gap between experimentation and theory is $13 \%$ (obtained for S0 at $2.3 \mathrm{MHz} \mathrm{mm}$ ). Fewer modes were measured with the same data compared with 2D-FFT processing. This is due to the temporal overlapping of modes. Even if the STFT process separates each frequency component, Lamb modes propagating together at the same frequency render the data

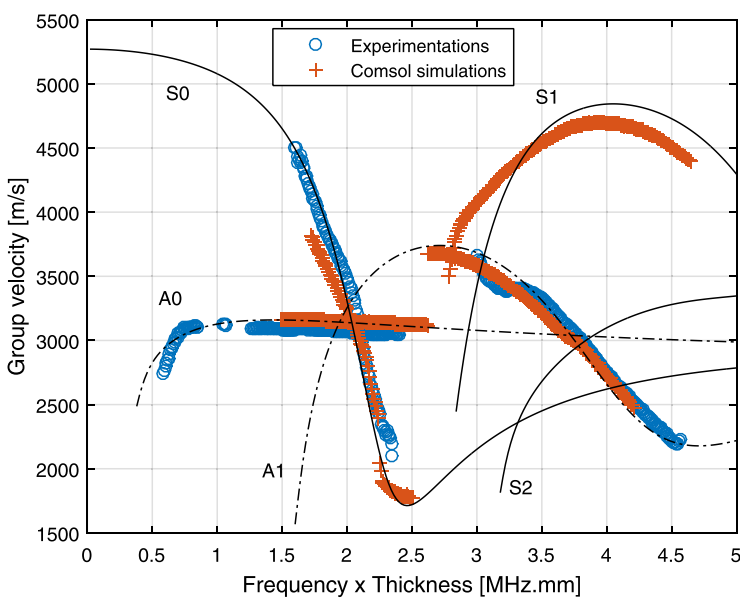

(b)

FIG. 5. (Color online) Measurement of (a) the phase velocity with 2D-FFT and (b) the group velocity using STFT. Comparison between theory (lines), FEM simulations (+), and experimental results (o). 


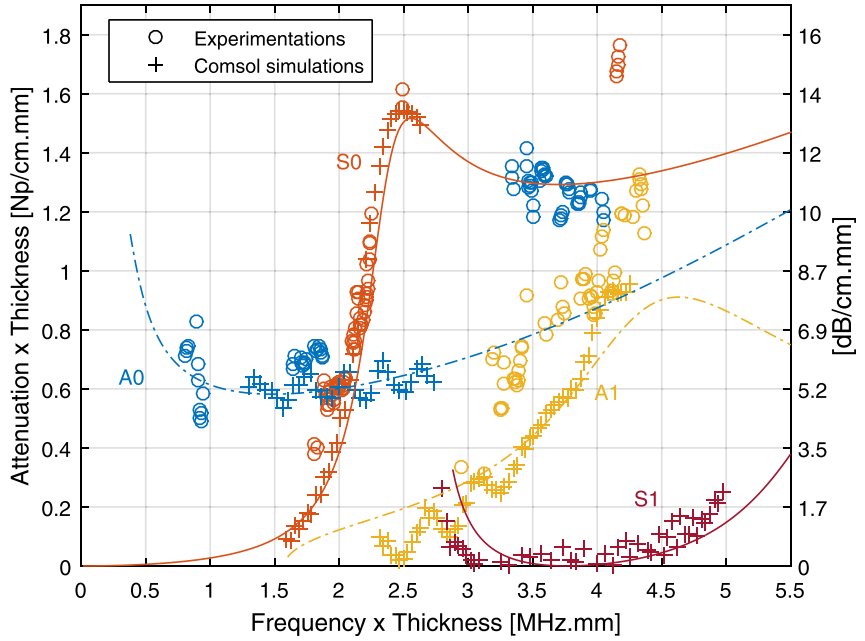

FIG. 6. (Color online) Measurement of leaky attenuation with 2D-FFT: comparison between the theory (lines), experimental results (o), and FEM simulations $(+)$. Attenuation is given in $\mathrm{Np} / \mathrm{cm}$ multiplied by the thickness of the plate in $\mathrm{mm}$.

unusable. The contribution of each Lamb mode has to be distinguished in order to calculate the velocity and attenuation. This is not always the case with this technique. In the case of two modes propagating together, the STFT measures an intermediate and meaningless value for the group velocity and attenuation.

However, when echoes are centered on different frequencies, this technique can be used to obtain a direct measurement of the group velocity while taking into account dispersive propagation as shown in Fig. 5(b). Good agreement with theory is thus achieved in this manner.

Based on the same experimental and computed data, the leaky attenuation was then measured, as shown in Fig. 6 for 2D-FFT processing and in Fig. 7 for STFT processing.

Each mode is identified with respect to their related phase or group velocity that was measured at the same time. There is a lack of experimental data for the S1 mode because its leaky attenuation is close to zero, which means that even if this mode is generated it will not reemit into the

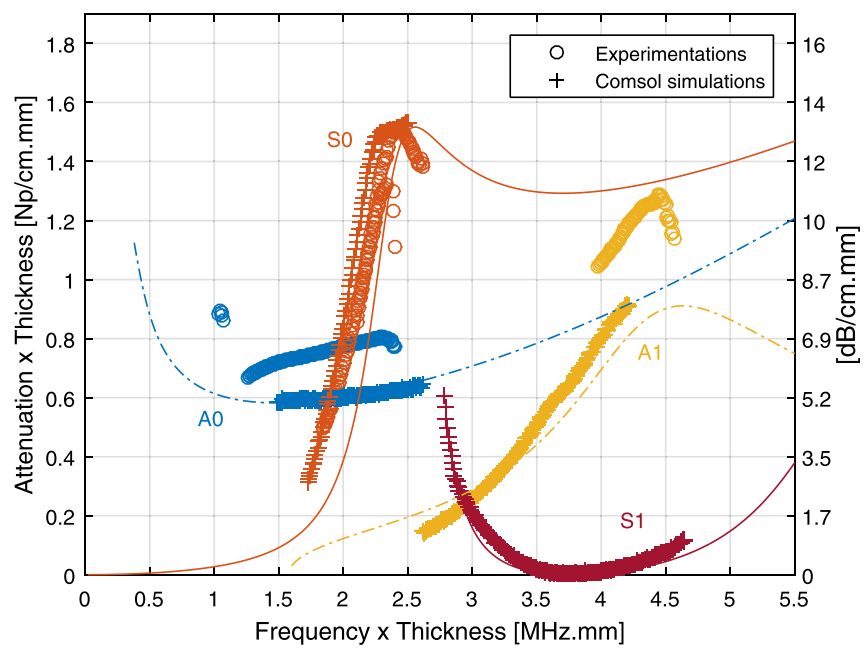

FIG. 7. (Color online) Measurement of leaky attenuation with STFT: comparison between theory (lines), experimental data (o), and FEM simulations (+). surrounding water. This behaviour was predicted theoretically in Pilarski et al. (1993). There is also a difference between the experimental results and theory. Experimental attenuation is generally higher than the theoretical value, especially from $3 \mathrm{MHz} \mathrm{mm}$ onwards: before $3 \mathrm{MHz} \mathrm{mm}$, the maximum gap is $20 \%$, and after it is between $33 \%$ and $45 \%$ for both processes. This gap is not seen with the attenuation results calculated on the basis FEM data. A possible assumption is that the material (e.g., absorption and scattering) has a non-negligible influence on attenuation when starting this frequency. However, this kind of attenuation would describe energy lost in heat, therefore it cannot be used to create and supply a new Lamb wave in a second parallel plate. This is why we focused on leaky attenuation in order to apply it to the two-plate case in Sec. III A.

Thus far, a study of leaky Lamb wave propagation has been given with respect to one immersed plate and dispersive propagation has been measured on the basis of experimental data (immersion in water). Phase and group velocities have been measured and compared to theory, which showed good agreement.

Attenuation in the case of an immersed plate has been theoretically calculated and experimentally measured with two different signal processing techniques. The implementation of these techniques has already been validated with FEM simulation. We found reasonably good agreement with theory. This attenuation characterizes the leakage of energy into the fluid and is central to our intention to propagate Lamb waves into other plates positioned further away from the first plate. Section III studies the propagation of leaky Lamb waves in a second plate.

\section{EXCITATION OF LEAKY LAMB WAVES IN A SECOND PARALLEL PLATE}

While Sec. II investigated the propagation of leaky Lamb modes in one plate, the generation of leaky Lamb modes in a second parallel and immersed plate is discussed in this section.

\section{A. Study of the immersed whole structure}

This section considers the two-plate system as a waveguide and computes its dispersion curves.

An immersed structure solid/fluid/solid (see Fig. 8) is now classically studied, with the interaction of a monochromatic oblique incident wave (angular frequency and incident angle, respectively, denoted $\omega$ and $\theta_{i}$ ) propagating in a semi-infinite fluid in the plane $(O x y)$ with the structure. The fluid media have all the same characteristics (celerity $c_{0}=1490 \mathrm{~m} / \mathrm{s}$, density $\rho_{0}=1000 \mathrm{~kg} / \mathrm{m}^{3}$, thickness $d_{p}=152.5 \mathrm{~mm}$ ) and the two isotropic solid plates are identical (celerity $c_{L}=5750 \mathrm{~m} / \mathrm{s}$ of the longitudinal waves, celerity $c_{T}=3150 \mathrm{~m} / \mathrm{s}$ of the transversal waves, density $\rho=7950 \mathrm{~kg} / \mathrm{m}^{3}$, thickness $h=7.8 \mathrm{~mm}$ ).

Contrary to the case particularly studied by Coulouvrat et al. (1998) the ratio of the thicknesses $h / d_{p}$ is much less than 1. The incident plane wave generates four waves in each solid layer and two waves in the fluid layer, which leads to 12 unknowns (included the reflected wave and the transmitted wave in the last fluid). The writing of the boundary 


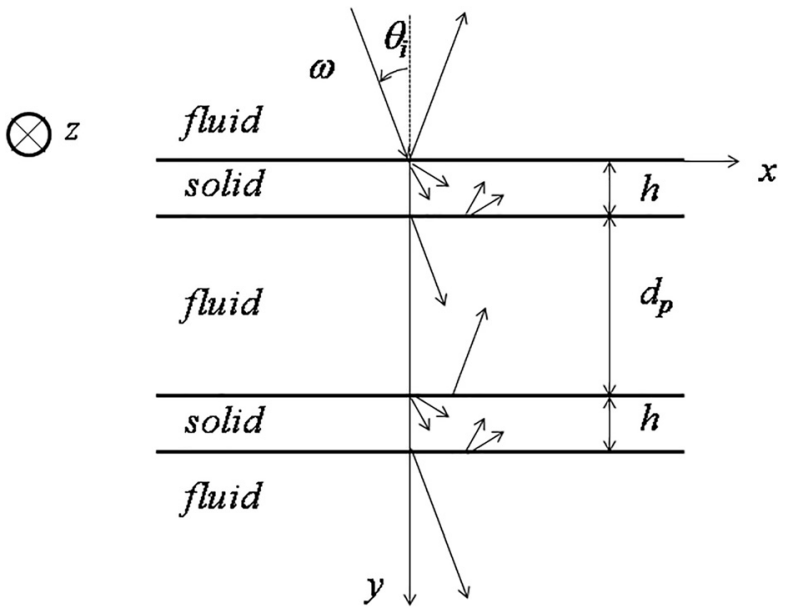

FIG. 8. Geometry of the problem: Interaction of an incident wave propagating in a fluid, with an immersed structure solid/fluid/solid.

conditions at each interface (equality of the normal displacement and of the stress vector) leads to 12 equations. In order to avoid any numerical problems due notably to the large thickness of the fluid layer, the reference of each plane wave is taken at the interface from where it propagates or decrease (Potel and de Belleval, 1993). A scanning of the reflection coefficient in the fluid permits to obtain the dispersion curves for the generalized Lamb modes for the whole structure (Potel et al., 1996) [Fig. 9(a)].

Several modes can be observed, which are highlighted by the superimposition of the Lamb modes for one of the solid plates (antisymmetric modes in blue and symmetric modes in red) and of the modes in a $d_{p}$-thick fluid guide with rigid walls [Fig. 9(b)]. The different modes $m$ in the fluid guide are given by

$$
k_{x_{m}}^{2}=\left(\frac{\omega}{c_{0}}\right)^{2}-\left(\frac{m \pi}{d_{p}}\right)^{2}, \quad \forall m \in \mathbb{N},
$$

where $k_{x_{m}}$ is the projection on the $x$-axis of the wave number vector of the $m$-th mode. Due to the large value of the thickness of the fluid layer, all the dispersion curves converge very rapidly towards the same curve given by $m=0$ (green line on Fig. 9). All the other black dispersion curves correspond to the interaction between all the reflections with the structure (coupling).

This proves that leaky Lamb modes that propagate in one plate will also propagate in two immersed plates and can potentially be used for NDT of both plates. We will now study the local attenuation of leaky Lamb wave in the second plate in order to get information on the feasibility of NDT in the second plate.

\section{B. The EBM}

This section describes a simple EBM in order to compute the Lamb mode amplitude created in a second parallel and immersed plate. Implications on the measurement methodology will be highlighted.

The system under investigation is composed of two parallel and immersed plates of the same thickness: a schematic view is given in Fig. 10(a). We assumed a steady state and a leaky Lamb mode propagating in the first plate, with attenuation due to the reemission of energy into the liquid: other attenuation mechanisms (such as absorption and scattering) were neglected. This leaky Lamb wave can be described by the amplitude of displacements along the $\mathrm{X}$ axis $U 1(x)$ $=U_{0} e^{-\alpha x}$, with $\alpha$ representing the leaky attenuation coefficient given by the theory for one immersed plate. This attenuation $\alpha$ depends on the mode selected, the frequency and the thickness of the plate (Figs. 6 or 7). The mechanical energy $E 1$ carried by this wave is proportional to the square of its amplitude, which means,

$$
E 1(x)=E_{0} e^{-2 \alpha x}
$$

with $E_{0}$ representing the initial energy at $x=0$.

We assumed that the plate radiated the same amount of energy on each side. This assumption is justified by simulations made in Hayashi and Inoue (2014): when a plate is loaded by water on only one side, its leaky attenuation is divided by 2 .

There is initially no propagating leaky Lamb mode in the second plate. However, the energy leaking from the leaky Lamb mode in the first plate creates a leaky Lamb mode in the second plate as shown by Corneloup et al.

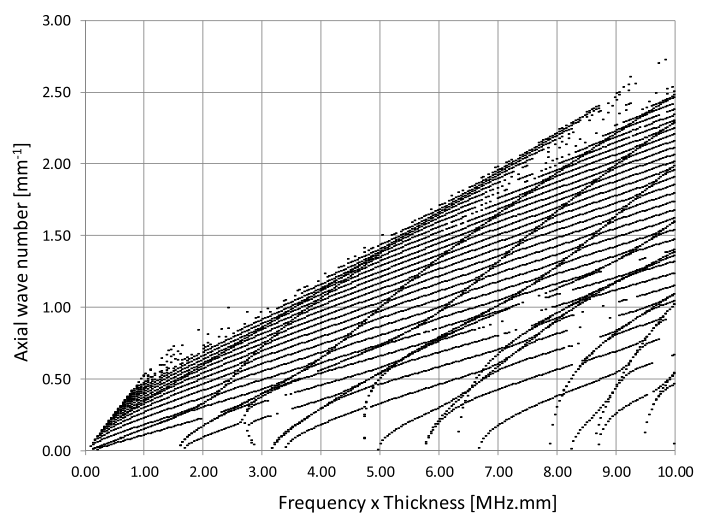

(a)

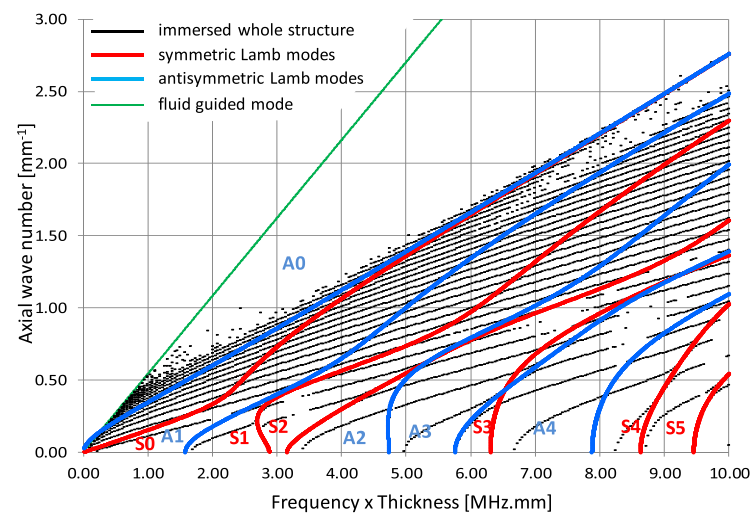

(b)

FIG. 9. (Color online) Dispersion curves of the immersed structure, in the plane $(f h, k x)$ (a). Superimposition (b) of the dispersion curves of one solid layer (red symmetric modes Sm and blue antisymmetric modes $\mathrm{Am}$ ), of the green dispersion curves of a fluid guide with perfectly rigid walls, and of the black dispersion curves of the whole immersed structure [see (a)], in the same plane. 


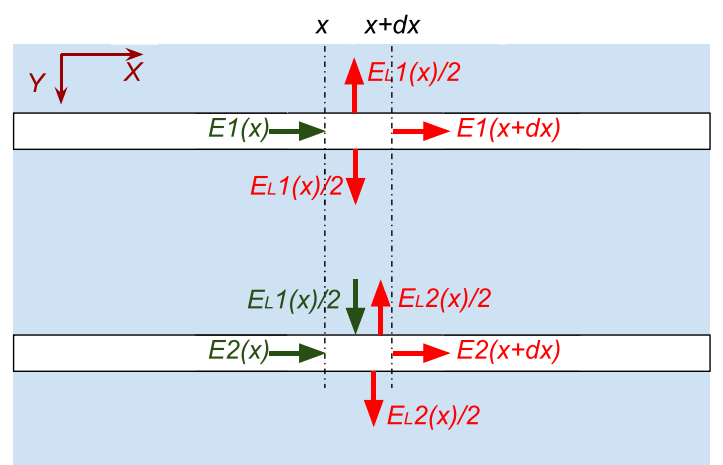

(a)

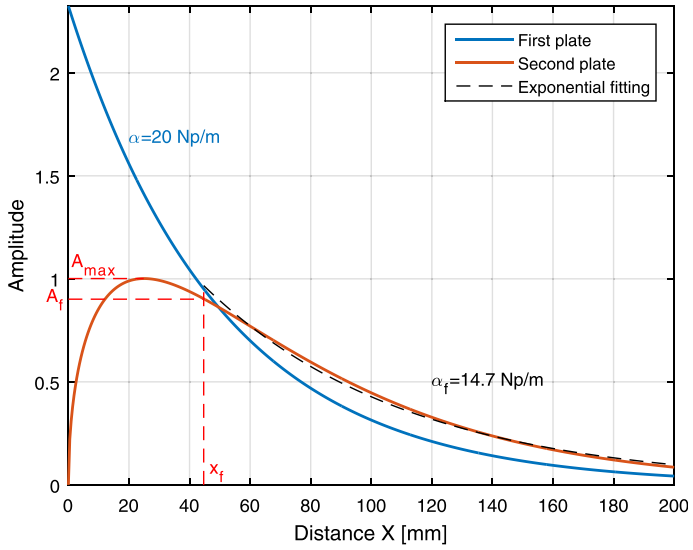

(b)

FIG. 10. (Color online) Energy balance in the two plates, with the gain written in green and the loss in red (a). Computed theoretical amplitude in the two plates with exponential fitting approximating the second plate profile decrease (b): the fit starts at $90 \%$ of the second plate's maximum amplitude.

(2011) and Schmitt et al. (2013). As the thicknesses of the plates are equal, the leaky Lamb modes are the same in the first and in the second plate. We assumed that all of the energy from the first plate supplied the leaky Lamb mode in the second plate, i.e., the full transmission of incident energy into the Lamb mode with no reflection and no direct transmission through the second plate. This is a key assumption because reflection and direct transmission through the second plate do occur. It nevertheless quantifies the incidence of the energy supply from the first plate to the second plate. The effects of other phenomena are highlighted by FEM in Sec. III C.

As illustrated in Fig. 10(a), an energy balance was established for a small portion of the first plate, between $x$ and $x+d x$. It gives

$$
E 1(x)=E 1(x+d x)+E_{L} 1(x)
$$

with $E_{L} 1(x)$ representing the energy that leaks into the fluid from the studied portion of plate. By combining Eq. (4) with Eq. (5), we were able to evaluate this quantity,

$$
E_{L} 1(x)=E 1(x)\left[1-e^{-2 \alpha d x}\right] .
$$

An energy balance was performed at the same abscissa in the second plate. It gives

$$
E 2(x)+\frac{1}{2} E_{L} 1(x)=E 2(x+d x)+E_{L} 2(x),
$$

with $E 2$ representing the mechanical energy carried by the leaky Lamb mode created in the second plate, and $E_{L} 2$ the amount of energy lost by leakage into the fluid. The contribution of the first plate is additive with the Lamb mode in the second plate because they are in phase. Leaky Lamb modes are identical in the two plates which makes it possible to evaluate $E_{L} 2$ by Eq. (6) with

$$
E_{L} 2(x)=E 2(x)\left[1-e^{-2 \alpha d x}\right] .
$$

Further operations give us an explicit expression to calculate the energy in the second plate,

$$
E 2(x+d x)=E 2(x) e^{-2 \alpha d x}+\frac{1}{2} E_{L} 1(x) .
$$

We computed the mechanical energy in the second plate and calculated the normalized amplitude by taking its square root. The results are plotted in amplitude in Fig. 10(b) for the case $\alpha=20 \mathrm{~Np} / \mathrm{m}$ (which corresponds to the maximum leaky attenuation of the S0 mode for a plate with a thickness of $7.8 \mathrm{~mm}$ ).

First of all, we were able to accurately calculate the amplitude on the first plate: it decreases exponentially with the correct attenuation $\alpha$. The amplitude on the second plate grows to reach a maximum before decreasing thereafter. However, because of the continuous supply from the first plate, the decrease is slower than in the first plate and no longer involves an exponential decrease.

This may be problematic because techniques to experimentally measure leaky attenuation are only valid if the decrease is exponential. In order to compare this theory with the results from experiments, we approximated the decrease in the second plate with an exponential fitting: it gives the apparent attenuation $\alpha_{f}$. This apparent attenuation is found systematically lower and proportional to attenuation in the case of one plate $\alpha$. However, the apparent attenuation depends on where the fit starts. Figure 10(b) shows an example with an exponential fitting using data located after a $10 \%$ decrease in the amplitude (i.e., $A_{f} / A_{\max }=0.9$ ); it gives an apparent attenuation of $14.7 \mathrm{~Np} / \mathrm{m}$ with an $R^{2}$ of 0.997 . This apparent attenuation would be equal to $16.5 \mathrm{~Np} / \mathrm{m}$ if we had started the fitting after a $50 \%$ decrease. Figure 11 shows the variation in the measurement as a function of the fitting start. It can be seen that the apparent attenuation fluctuates greatly: it tends to $0.68 \alpha$ for a measurement starting at the amplitude maximum and to $0.94 \alpha$ if the measurement is started at the end of propagation. This could be a systematic error for measuring experimental attenuation on a system of two plates.

To overcome this bias, measurements were systematically performed after a $10 \%$ decrease in amplitude on the second plate: this rule appears to be an acceptable compromise between the range of validity of this apparent attenuation and the quality of the fitting. The measured attenuation 


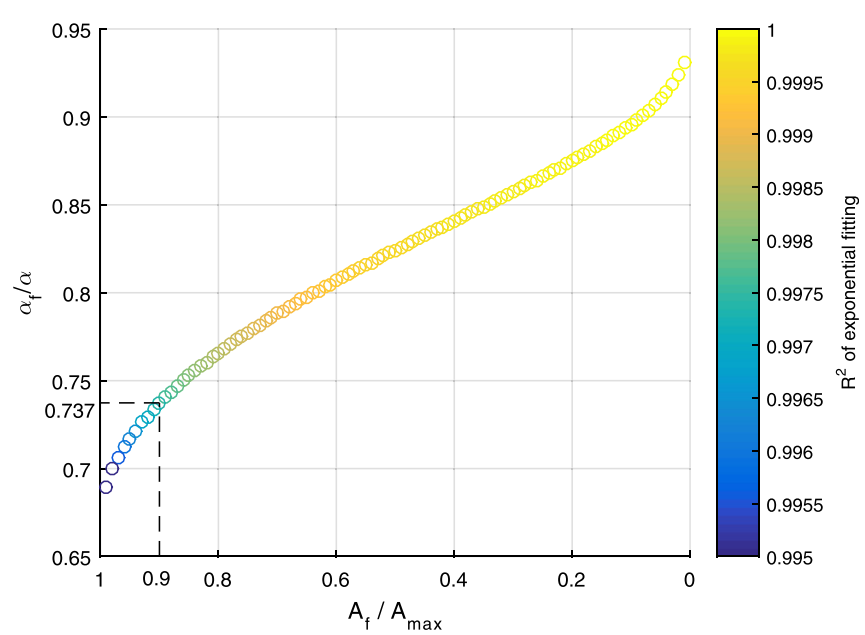

FIG. 11. (Color online) Theoretical apparent leaky attenuation $\left(\alpha_{f}\right)$ normalized by the leaky attenuation in the case of one plate $(\alpha)$ as a function of the amplitude in the second plate where the fit starts $\left(A_{f}\right)$, normalized by the maximum amplitude in the second plate $\left(A_{\max }\right) \cdot R^{2}$ of exponential fitting is plotted in the color scale.

in the second plate is therefore expected to be $0.737 \alpha$ for each leaky Lamb mode studied. This is the theoretical value plotted in Fig. 18 and compared with the experimental measurement and simulations.

\section{Finite-element simulations}

This section presents the FEM model used in the frequency domain to investigate the propagation of leaky Lamb modes from the first plate into the second plate. Areas that are insonified in the two plates system are located. The theory developed in Sec. III B for the second plate is compared with the simulation results in order to evaluate limits of this theory.

\section{Presentation of two-plates simulations}

The previous part has shown that the transducer can be used to generate multiple leaky Lamb modes (Fig. 4). This has been taken into account by the use of two postprocessing techniques (2D-FFT and STFT). However, for simulations in the frequency domain, multi-modal propagation renders simulation useless. To avoid this, the incident beam produced by the simulated transducer was optimized. In order to increase the directivity of its main beam, its diameter was widened $(200 \mathrm{~mm}$ instead of 38.1 to $50 \mathrm{~mm}$ for experiments). The prescribed pressure was windowed by a Gaussian function in order to render the side lobes negligible. The distance between plates was widened in order to clearly separate the successive reemission of each plate.

The results are shown in Fig. 12 for the studied configuration. The incident acoustic beam has excellent directivity and no observable side lobes as planned. Consequently, it excites a unique leaky Lamb mode (A1) in the first plate. This mode is transmitted to the second plate. A zoom is shown in Fig. 12(b) to show the vertical displacement of A1 modes generated in the second plate. The phase velocity has

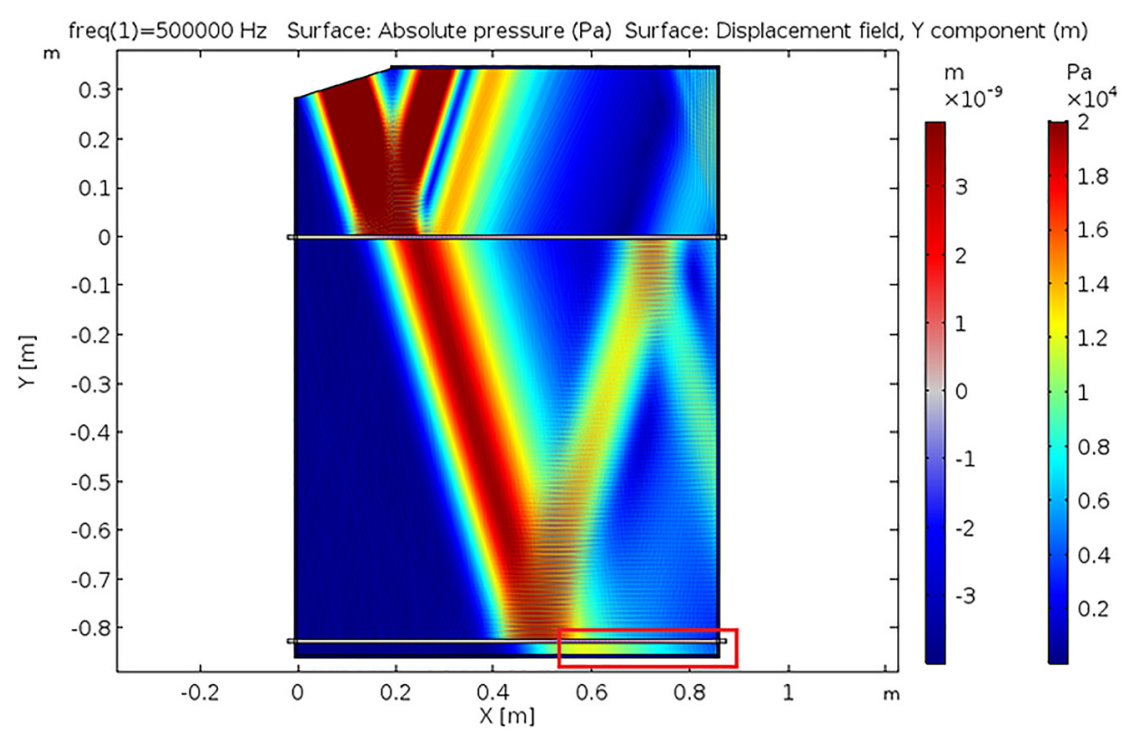

(a)

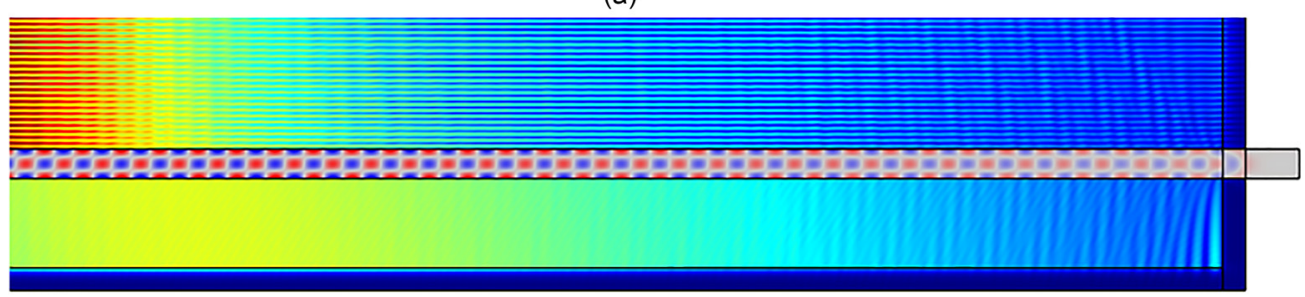

(b)

FIG. 12. (Color online) FEM in the frequency domain of two immersed and parallel plates excited with an optimized acoustic beam for the studied configuration (a), zoom on the red rectangle showing vertical displacement of the A1 leaky Lamb mode (b). 


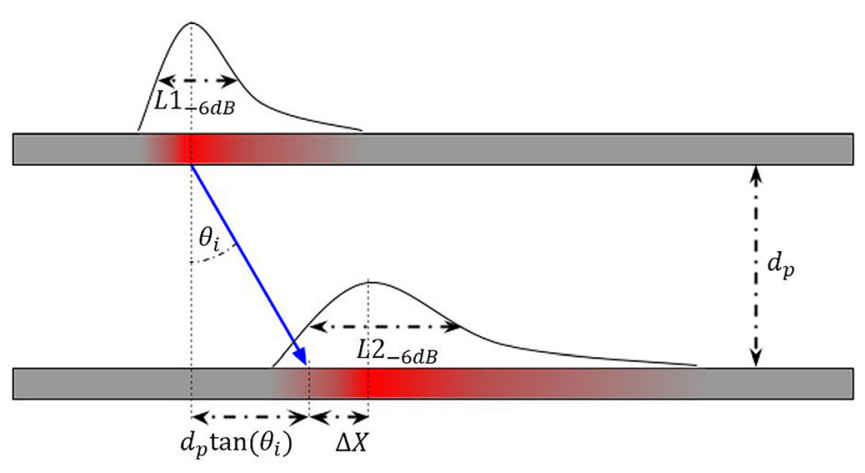

FIG. 13. (Color online) Schematization of the two plates system with denomination of insonified areas.

been measured and confirmed the identification of the A1 mode in the first and the second plate.

We will now investigate the location and the shape of theses different amplitudes profiles.

\section{Localization of insonified areas}

As seen in Fig. 12, the propagation of leaky Lamb wave in two parallel plates induce unequal amplitudes into the plates. The incident beam gives the initial amplitude in the first plate and the amplitude decreases quickly because of energy leakage into the fluid. There is then a maximum of amplitude, which is the best place to perform NDT. The reemission of the first plate create a new acoustic beam incident on the second plate, which lead to a new maximum of amplitude into the second plate. The offset between the locations of these two maxima can be decompose in two part: the geometrical offset and the remaining part which will be called the leaky offset. The geometrical offset is due to the reemission angle $\theta_{i}$ and is equal to $d_{p} \tan \left(\theta_{i}\right)$, with $d_{p}$ the distance between plates. The leaky offset $\Delta X$ is caused by the alimentation and the continuous reemission of energy in the second plate: there is no analytical formulae to calculate it; it has to be computed by FEM or EBM. We will also measure the plate length where the amplitude is higher than $-6 \mathrm{~dB}$ around the maximum of the first plate $\left(L 1_{-6 \mathrm{~dB}}\right)$ and around

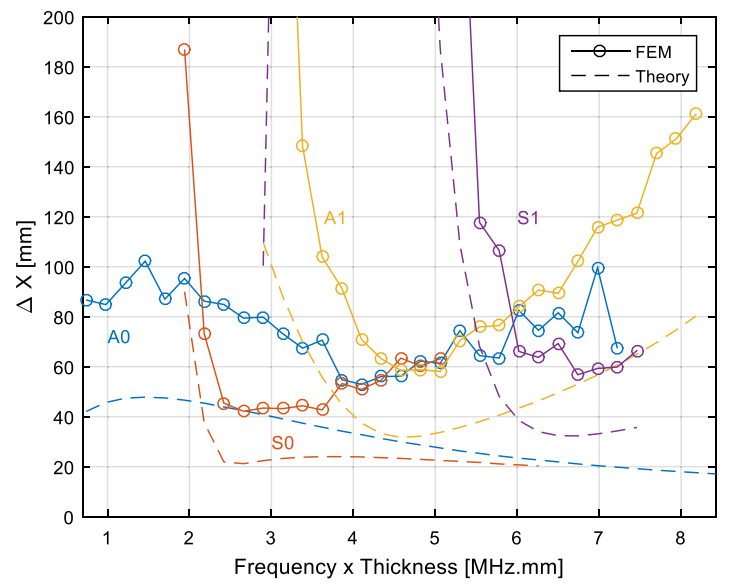

(a) the maximum of the second plate $\left(L 2_{-6 \mathrm{~dB}}\right)$. Figure 13 schematizes these notations.

We will study these three parameters $\left(\Delta X, L 1_{-6 \mathrm{~dB}}\right.$, and $L 2_{-6 \mathrm{~dB}}$ ) using FEM and EBM. FEM simulations were computed for A0, S0, A1, and S1 leaky Lamb modes in a large frequency range (from 1 to $9 \mathrm{MHz} \mathrm{mm}$ ). We extracted the amplitude from both plates, and measured the leaky offset $\Delta X$ and the insonification lengths $L 1_{-6 \mathrm{~dB}}$ and $L 2_{-6 \mathrm{~dB}}$ for each case.

Figure 14 shows the leaky offset $\Delta X$ for the first four leaky Lamb modes with the optimized transducer used for FEM. In Fig. 14(a), we can see that the EBM models well variations of the leaky offset for each modes. However, EBM predictions of $\Delta X$ are systematically smaller by $45 \%$ $( \pm 5 \%)$ than FEM, which is the more reliable method.

Both methods are showing that leaky offset is strongly dependent on the leaky attenuation coefficient. Figure 14(b) shows the same data plotted in function of the attenuation coefficient: higher leaky offset are achieved for smaller leaky attenuation. The same behavior is also observed for $L 1_{-6 \mathrm{~dB}}$ and $L 2_{-6 \mathrm{~dB}}$ that are directly plotted in function of leaky attenuation in Fig. 15. The length of insonification is always larger in the second plate than in the first plate. All of this means that the amplitude profile is "flattening" or "smoothening" when it comes to the next plates, especially for a low leaky attenuation coefficient.

This "flattening" will be illustrated in the Fig. 16 where amplitudes from both plates are plotted. We will now investigate the rate of decreasing in the second plate and limitations of the EBM.

\section{Study of the decrease in the second plate}

The amplitude in the first and second plate were extracted and compared in Fig. 16 with the theoretical results from EBM. The amplitude from the first plate was scaled to be compared with the EBM, and the amplitude from the second plate was scaled by the same factor. The abscissas of both curves were shifted according to the maximum of the first plate. For the second plate, we choose to plot the abscissa without the geometrical offset $\left[d_{p} \tan \left(\theta_{i}\right)\right]$ for a

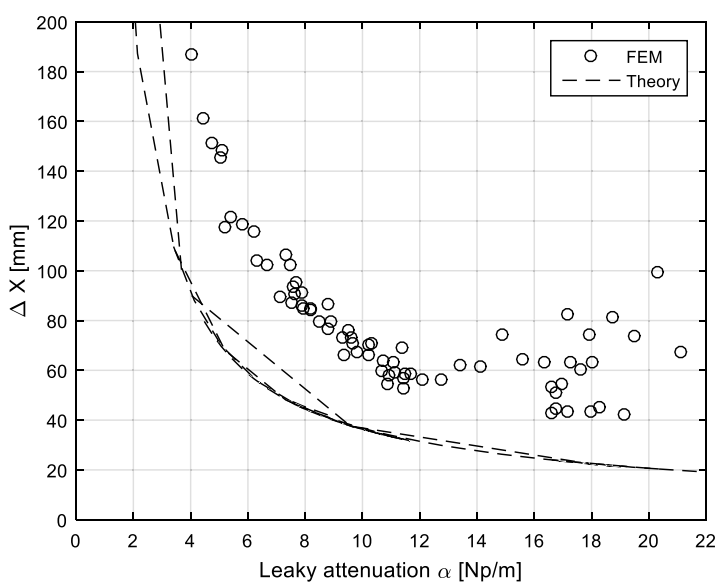

(b)

FIG. 14. (Color online) Leaky offset in second plate plotted in function of frequency-thickness (a) and leaky attenuation coefficient (b): FEM (o) and EBM (dashed lines). 


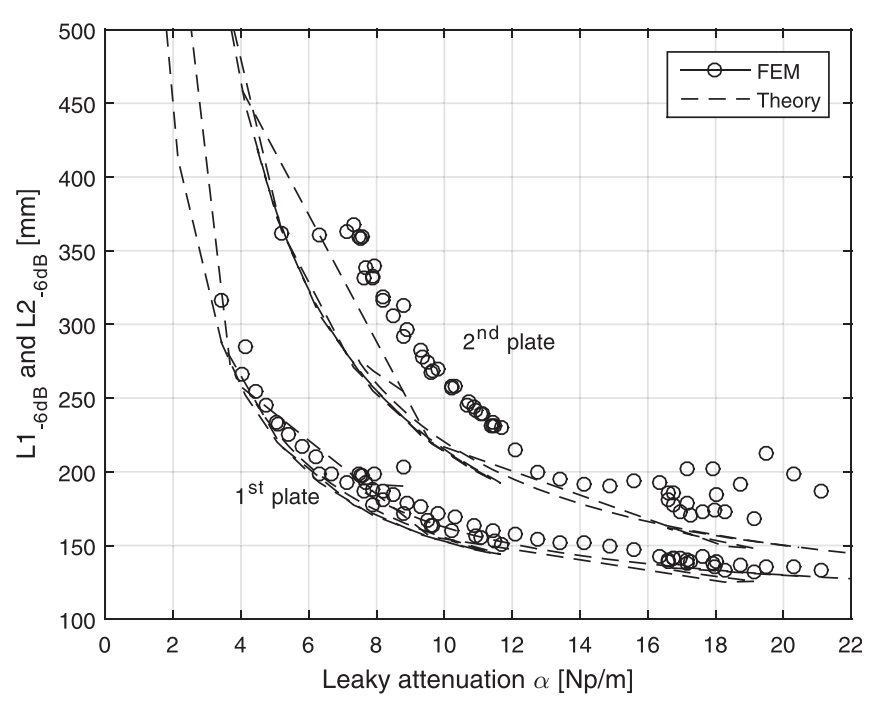

FIG. 15. Length of insonification at $-6 \mathrm{~dB}$ in the first plate and the second plate for FEM (o) and EBM (dashed lines) plotted in function of the leaky attenuation.

better visualization of energy exchanges. No further operation was performed to observe the limitations of the theory.

As for Figs. 14 and 15, we adapted the EBM so the leaky Lamb wave in the first plate would be generated by an incident amplitude with the same Gaussian profile as that for FEM. We applied the same assumption as that used for the second plate, i.e., there is no direct reflection or throughplate transmission. The effect of Gaussian excitation on apparent attenuation was evaluated and found negligible: $6.023 \mathrm{~Np} / \mathrm{m}$ without Gaussian excitation (see Fig. 10) and $6.017 \mathrm{~Np} / \mathrm{m}$ with Gaussian excitation (see Fig. 16).

The theoretical and simulated amplitudes for the first plate are in excellent agreement. For the second plate, the theory is less accurate but still predicts the tendency of the amplitude. The maximum amplitude in the second plate is predicted with a $5 \%$ error, which is remarkable regardless of the strong assumption on direct transmission and reflection. Apparent attenuations measured after a $10 \%$ decrease in the second plate are quite similar: $6.0 \mathrm{~Np} / \mathrm{m}$ for the EBM, 5.0 for FEM (compared with the one plate attenuation of $8.2 \mathrm{~Np} / \mathrm{m}$ ).

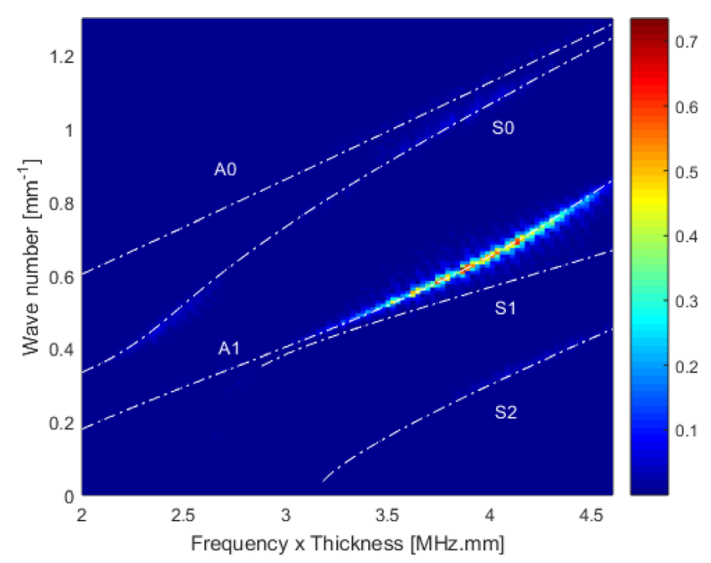

(a)

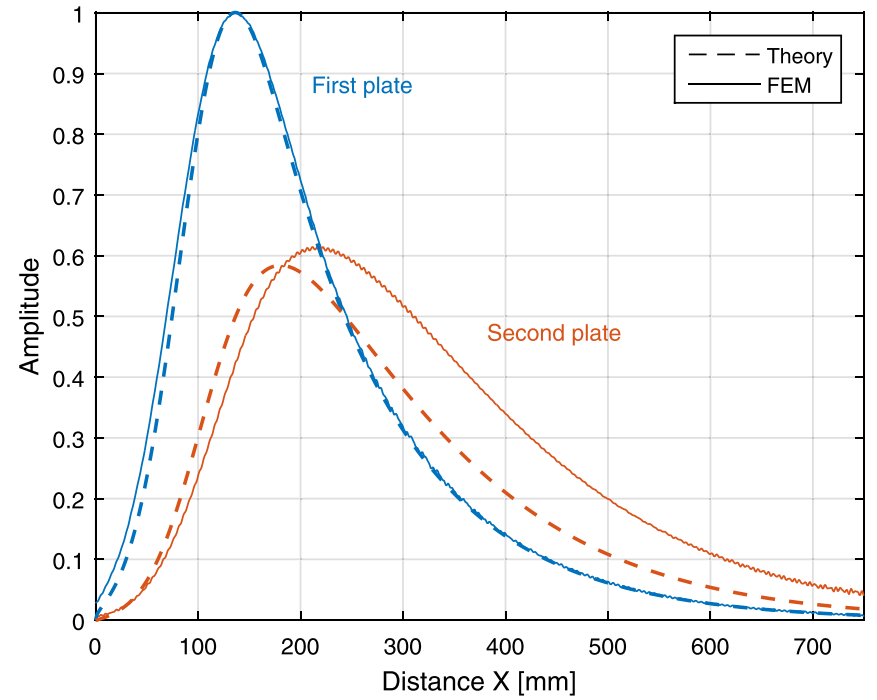

FIG. 16. (Color online) Comparison of the amplitude between theory (EBM) and FEM in two parallel plates.

Energy exchanges between plates appear to have a predominant effect on the creation of a leaky Lamb wave in the second plate. Other physical phenomena (such as direct reflection or through-plate transmission) have a weaker impact on the amplitude of the Leaky Lamb mode created in the second plate.

The phase has been extracted in the second plate as a function of the distance, and the phase velocity was computed by a linear regression. We obtained an excellent fitting, with an $R^{2}$ regression superior to 0.999 . The leaky attenuation was calculated by an exponential fitting of the amplitude as a function of the distance, with data selected after a $10 \%$ decrease in the amplitude. The phase velocity and leaky attenuation are plotted in Figs. 17 and 18, respectively.

\section{Experimentation}

Experiments were conducted with the same protocol as that described in Sec. II C, except that the hydrophone scanned the reemitted field after the second plate. This

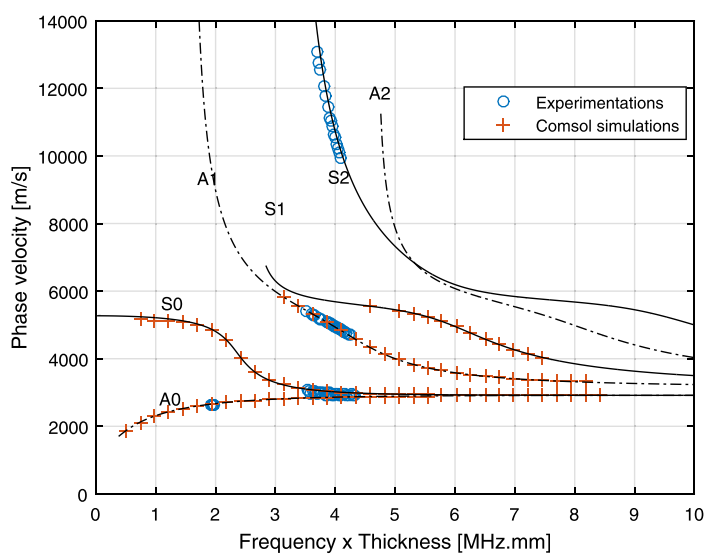

(b)

FIG. 17. (Color online) Measurement of the phase velocity in the second plate: (a) experimental 2D-FFT of the reemitted field after the second plate in the studied configuration (theoretical wave numbers are plotted in dotted-dash lines) and (b) comparison between the theoretical phase velocity (lines), FEM simulations (+) and experimental data measured with 2D-FFT (o). 


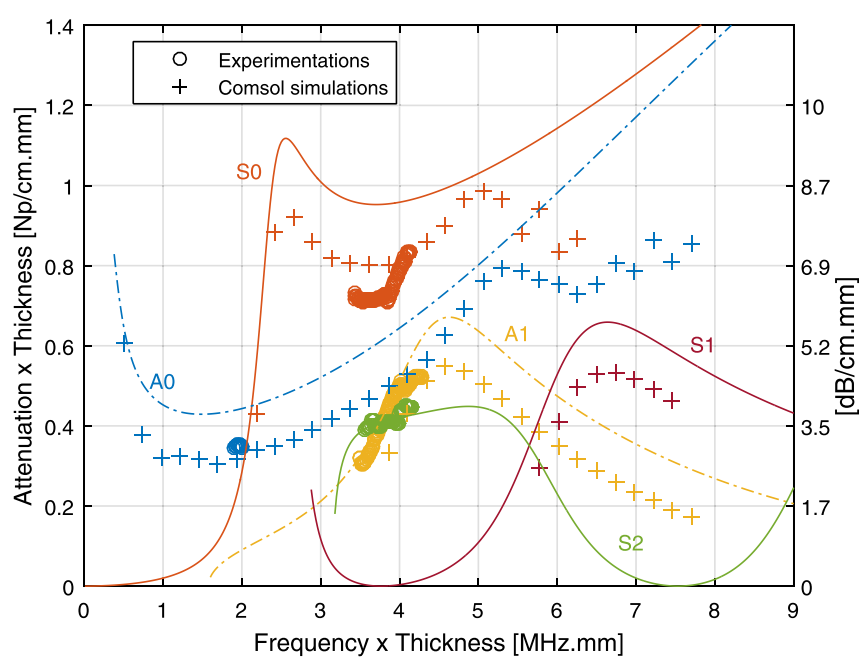

FIG. 18. (Color online) Measurement of leaky attenuation in the second plate: comparison between the EBM data for two plates (lines), FEM simulations $(+)$, and experimental data measured with STFT (o). Attenuation is expressed in $\mathrm{Np} / \mathrm{cm}$ multiplied by the plate thickness in $\mathrm{mm}$.

second plate had the same thickness as the first plate $(7.8 \mathrm{~mm})$ and was positioned parallel to it. They were separated by $152.5 \mathrm{~mm}$ of water.

The phase velocity was calculated with 2D-FFT processing, while attenuation was calculated with STFT (we fitted the data after a $10 \%$ decrease in the amplitude). The attenuation in the second plate was not measured by 2D-FFT because the calculation would be based on only two points and the assumption that the decrease is exponential (Castaings et al., 2004). Since the expected profile is no longer an exponential decrease in the second plate, 2D-FFT is no longer relevant to measure attenuation. The STFT is more reliable in this case because it uses an exponential fitting based on an all set of points.

The results are plotted against the theoretical and simulation results and discussed in Fig. 18.

\section{E. Results and discussion}

The experimental 2D-FFT for the studied configuration is plotted Fig. 17(a): the A1 leaky Lamb mode propagates in the second plate. The phase velocity was calculated using wavenumbers and frequencies of maximum amplitude, which are plotted in Fig. 17(b). We observed excellent agreement between COMSOL Multiphysics ${ }^{\circledR}$ simulation, experimentation, and theory: when a leaky Lamb mode is reemitted from the first plate, it is generated into the second plate.

Leaky Lamb modes propagate in the second plate with the same velocity (phase and group) as that in the first plate. However, the second plate is continuously sustained by the acoustic reemission of the first plate as seen in the EBM (Fig. 10) and in simulation (Fig. 12). The amplitude decreases, but at a slower rate than in the first plate: the apparent leaky attenuation is always inferior to the leaky attenuation for one plate. The EBM, simulation and experimental results are in good agreement as shown in Fig. 18.

\section{CONCLUSION}

Leaky Lamb waves were studied in the first part. Techniques to measure their properties (phase and group velocity, leaky attenuation) were validated by comparison between the theoretical, experimental, and simulation data. Short-time Fourier transform and 2D-FFT were applied to the two-plate case in Sec. III.

For two plates of the same thickness, dispersion curves have been computed showing the propagation of leaky Lamb modes in both plates. More locally, a focus has been made on the transmission of leaky Lamb modes from the first plate to the second plate in order to investigate on the NDT of the second plate. Due to the reemitted field from the first plate, leaky attenuation in the second plate is much weaker than for the one-plate case, and it has been successfully measured by the STFT technique. We proposed a simple EBM to quantify the energy supply from the first plate to the second plate, and this model proved to be accurate enough to predict the leaky Lamb wave amplitude and apparent attenuation in the second plate.

Now that the propagation of leaky Lamb waves in a second plate is established, the next step is to identify parameters of interest for NDT in the second plate and to optimize them. Echoes from both plates will be located with their time of flight and their amplitude. Echoes from the second plate will be delayed because of the fluid layer, which imposes a propagation speed that is usually smaller than the group velocity of Lamb waves. With the EBM we quantified the amplitude in the second plate, which proved to be smaller than that in the first plate: this will also help us locate default in plates, even if calibration against a controlled default in the first plate may be necessary. It is important to stress the fact that this methodology is applicable to more parallel plates, thus opening the door to the possibility of inspecting three or four parallel plates. Experimental detections in a set of two and three parallel plates and comparison with the EBM has been presented by Kauffmann et al. (2018a). We have shown that NDT of the third plate is possible. Results will be available in the corresponding proceedings.

\section{ACKNOWLEDGMENTS}

This research was supported by the CEA Cadarache centre, France.

Alleyne, D., and Cawley, P. (1991). "A two-dimensional Fourier transform method for the measurement of propagating multimode signals," J. Acoust. Soc. Am. 89, 1159-1168.

Alleyne, D., and Cawley, P. (1992a). "Optimization of Lamb wave inspection techniques," NDT E Int. 25, 11-22.

Alleyne, D., and Cawley, P. (1992b). "The interaction of Lamb waves with defects," IEEE Trans. Ultrason. Ferroelectr. Freq. Control 39, 381-397.

Baqué, F., Jadot, F., Marlier, R., Saillant, J. F., and Delalande, V. (2015). "In service inspection and repair of the sodium cooled ASTRID reactor prototype," presented at the International Congress on Advances in Nuclear Power Plants (ICAPP'2015), Nice, France.

Baqué, F., Paumel, K., Corneloup, G., Ploix, M. A., and Augem, J. M. (2011). "Non destructive examination of immersed structures within liquid sodium," in 2011 2nd International Conference on Advancements in Nuclear Instrumentation, Measurement Methods and their Applications, (ANIMMA), Ghent, Belgium. 
Baqué, F., Reverdy, F., Augem, J.-M., and Sibilo, J. (2012). "Development of tools, instrumentation and codes for improving periodic examination and repair of SFRs," Sci. Technol. Nucl. Install. 2012, 718034.

Bertoni, H. L., and Tamir, T. (1973). "Unified theory of Rayleigh-angle phenomena for acoustic beams at liquid-solid interfaces," Appl. Phys. 2, $157-172$.

Castaings, M., Bacon, C., Hosten, B., and Predoi, M. V. (2004). "Finite element predictions for the dynamic response of thermo-viscoelastic material structures," J. Acoust. Soc. Am. 115, 1125-1133.

Castaings, M., Le Clezio, E., and Hosten, B. (2002). "Modal decomposition method for modeling the interaction of Lamb waves with cracks," J. Acoust. Soc. Am. 112, 2567-2582.

Chan, C. W., and Cawley, P. (1998). "Lamb waves in highly attenuative plastic plates," J. Acoust. Soc. Am. 104, 874-881.

Chimenti, D. E., and Rokhlin, S. I. (1990). "Relationship between leaky Lamb modes and reflection coefficient zeroes for a fluid-coupled elastic layer,” J. Acoust. Soc. Am. 88, 1603-1611.

Coquin, G. A. (1964). "Attenuation of guided waves in isotropic viscoelastic materials," J. Acoust. Soc. Am. 36, 1074-1080.

Corneloup, G., Ploix, M.-A., Chaix, J.-F., Lillamand, I., and Baqué, F. (2011). "Potential of ultrasounds for NDT of a structure located behind parallel immersed plates," AIP Conf. Proc. 1335, 1695-1700.

Coulouvrat, F., Rousseau, M., Lenoir, O., and Izbicki, J. L. (1998). "Lambtype waves in a symmetric solid-fluid-solid trilayer," Acustica 84, 12-20.

Dayal, V., and Kinra, V. K. (1989). "Leaky Lamb waves in an anisotropic plate I: An exact solution and experiments," J. Acoust. Soc. Am. 85, 2268-2276.

Harb, M. S., and Yuan, F. G. (2015). "A rapid, fully non-contact, hybrid system for generating Lamb wave dispersion curves," Ultrasonics 61, 62-70.

Hayashi, T., and Inoue, D. (2014). "Calculation of leaky Lamb waves with a semi-analytical finite element method," Ultrasonics 54, 1460-1469.

Kauffmann, P., Ploix, M.-A., Chaix, J.-F., Gueudré, C., Corneloup, G., and Baqué, F. (2018a). "Non-destructive testing of nuclear structures behind screen using leaky Lamb waves," in 45th Annual Review of Progress in Quantitative Nondestructive Evaluation (QNDE 2018), Burlington, Vermont.

Kauffmann, P., Ploix, M.-A., Chaix, J.-F., Gueudré, C., Corneloup, G., and Baqué, F. (2018b). "Study of Lamb waves for non-destructive testing behind screens," in ANIMMA 2017-Advancements in Nuclear Instrumentation Measurement Methods and their Applications, Liège, Belgium.

Kobayashi, K. (1994). "Relationship between speed of Lamb wave and measurement error on distance,” Jpn. J. Appl. Phys. 33, 3115-3120.

Lindner, G., Faustmann, H., Frankenberger, S., Munch, M., Pflaum, K., Rothballer, S., and Unterburger, M. (2006). "Versatile acoustic waveguide sensor for liquids based on multiple mode conversion at solid-liquid interfaces," IEEE Ultrason. Symp. 2006, 1181-1184.

Luis Dean-Ben, X., Trillo, C., Doval, A. F., and Fernandez, J. L. (2010). "Phase and group velocity measurement of ultrasonic guided wavetrains in plates by pulsed TV holography," J. Acoust. Soc. Am. 127, 2210-2219.

Merkulov, L. G. (1964). "Damping of normal modes in a plate immersed in a liquid," Sov. Phys. Acoust. 10, 169-173.
Minonzio, J.-G., Foiret, J., Talmant, M., and Laugier, P. (2011). "Impact of attenuation on guided mode wavenumber measurement in axial transmission on bone mimicking plates," J. Acoust. Soc. Am. 130, 3574-3582.

Nayfeh, A. H. (1995). Wave Propagation In Layered Anisotropic Media: With Applications to Composites, North-Holland Series in Applied Mathematics and Mechanics (Elsevier, Amsterdam).

Nayfeh, A. H., and Nagy, P. B. (1997). "Excess attenuation of leaky Lamb waves due to viscous fluid loading," J. Acoust. Soc. Am. 101, 2649-2658.

Niethammer, M., Jacobs, L. J., Qu, J., and Jarzynski, J. (2000). "Time-frequency representation of Lamb waves using the reassigned spectrogram," J. Acoust. Soc. Am. 107, L19-L24.

Paget, C. A., and Rehman, M. A. (2017). "Multimodal location algorithm for Lamb waves propagating through anisotropic materials," in Proceedings of Sensors and Smart Structures Technologies for Civil, Mechanical, and Aerospace Systems 2017, edited by J. P. Lynch (SPIE, Bellingham).

Pavlakovic, B., Lowe, M., Alleyne, D., and Cawley, P. (1997). "Disperse: A general purpose program for creating dispersion curves," in Review of Progress in Quantitative Nondestructive Evaluation, edited by D. O. Thompson and D. E. Chimenti (Springer, New York), pp. 185-192.

Pilarski, A., Ditri, J. J., and Rose, J. L. (1993). "Remarks on symmetric Lamb waves with dominant longitudinal displacements," J. Acoust. Soc. Am. 93, 2228-2230.

Potel, C., and de Belleval, J.-F. (1993). "Acoustic propagation in anisotropic periodically multilayered media: A method to solve numerical instabilities," J. Appl. Phys. 74, 2208-2215.

Potel, C., de Belleval, J.-F., Genay, E., and Gatignol, P. (1996). "Behavior of Lamb waves and multilayered Rayleigh waves in an anisotropic periodically multilayered medium application to the long-wave length domain," Acust. Acta Acust. 82, 738-748.

Rose, J. L. (1999). Ultrasonic Waves in Solid Media (Cambridge University Press, Cambridge).

Schmitt, M., Schmidt, K., Olfert, S., Rautenberg, J., Lindner, G., Henning, B., and Reindl, L. M. (2013). "Detection of coatings within liquid-filled tubes and containers by mode conversion of leaky Lamb waves," J. Sens. Sens. Syst. 2, 73-84.

Takiy, A. E., Kitano, C., Higuti, R. T., Granja, S. C. G., Prado, V. T., Elvira, L., and Martinez-Graullera, O. (2017). "Ultrasound imaging of immersed plates using high-order Lamb modes at their low attenuation frequency bands," Mech. Syst. Signal Process. 96, 321-332.

Viktorov, I. A. (2013). Rayleigh and Lamb Waves: Physical Theory and Applications, softcover reprint of the original, 1st ed. (Springer, Berlin).

Wilcox, P. D. (2003). "A rapid signal processing technique to remove the effect of dispersion from guided wave signals," IEEE Trans. Ultrason. Ferroelectr. Freq. Control 50, 419-427.

Wilcox, P. D., Lowe, M. J. S., and Cawley, P. (2001a). "Mode and transducer selection for long range Lamb wave inspection," J. Intell. Mater. Syst. Struct. 12, 553-565.

Wilcox, P. D., Lowe, M. J. S., and Cawley, P. (2001b). "The effect of dispersion on long-range inspection using ultrasonic guided waves," NDT E Int. 34, 1-9.

Xu, Y.-F., and Hu, W.-X. (2017). "Wideband dispersion removal and mode separation of Lamb waves based on two-component laser interferometer measurement," Chin. Phys. B 26, 094301. 